\title{
Impact Analysis of SBAS Authentication
}

Ignacio Fernández-Hernández (EC), E. Châtre (EC), Andrea Dalla Chiara (Qascom), Giacomo Da Broi (Qascom), Oscar Pozzobon (Qascom), Javier Fidalgo (GMV), Miguel Odriozola (GMV), Ginés Moreno (GMV), Silvia Sturaro (University of Padua), Gianluca Caparra (University of Padua), Nicola Laurenti (University of Padua), Vincent Rijmen (IMEC-COSIC, KU Leuven)

\begin{abstract}
The GNSS community is dedicating significant effort to protect applications from harmful interference, including spoofing. As part of this effort, Europe will authenticate the Galileo Open Service message and is currently evaluating authentication schemes for EGNOS. This article presents the main results of recent EU activities on EGNOS authentication. After presenting some examples that illustrate the importance of authenticating SBAS, this article describes the main drivers for SBAS authentication design, namely: the use of I and/or Q channels, the use of the L1 and/or L5 frequencies, the authentication latency, and the cryptographic parameters. Later, the article presents the performances of EC-Schnorr and TESLA data authentication schemes, and analyses their impact in the SBAS L1 message sequence and in the L1/L5 Dual Frequency MultiConstellation Standard message sequence, currently under development.
\end{abstract}

\section{INTRODUCTION}

It is common knowledge that GNSS signals are received on the ground with a very low power level, and this makes them vulnerable to natural and artificial electromagnetic phenomena that can degrade or disrupt the service. Unprotected receivers can easily misinterpret signals from repeaters, test-beds or malicious attackers as real GNSS signals with severe consequences, especially for safety-of-life applications. Fortunately, there has yet to be a life threatening aircraft incident due to counterfeit GNSS signals. However, there have been cases of service denial due to jamming from personal privacy devices (PPDs) (FAA Navigation Team, 2011) and false aircraft positions reported due to GPS repeaters (Hünerbein \& Lange, 2015). While spoofers are not widespread yet, they represent a threat, particularly thanks to the popularization of software-defined radio (SDR) technologies. An aircraft's GNSS receiver without any protection could be spoofed by a nearby vehicle carrying a simple spoofer, even if the aircraft is not the intended victim. Similarly, satellitebased test-beds inadvertently transmitting GNSS/SBAS-like signals with operational PRNs could put at risk receivers within an area as big as the transmitting satellite footprint.

GNSS/SBAS can protect its data through message authentication. GNSS message authentication has been studied for GPS (Wesson, Rothlisberger, \& Humphreys, 2012), Galileo (FernándezHernández, et al., 2016) and Beidou (Yuan, Lv, Chen, Li, \& \& Ou, 2017), although, at the time of writing, only Galileo has confirmed its implementation (European Commission , 2017). GNSS signals may also incorporate spreading code authentication (Scott, 2003) and encryption features, such as those in Galileo Commercial Service (Calle, et al., 2016), Public Regulated Service, or GPS $\mathrm{P}(\mathrm{Y})$ and $\mathrm{M}$ codes, which protect against spoofing of the pseudorange measurements. However, some threats, such as meaconers or GPS repeaters, need to be treated in the receiver, by monitoring AGC, $\mathrm{C} / \mathrm{N}_{0}$, clock, signal correlation shape, PVT output, or adding antenna arrays or INS. Eventually, the highest degree of resilience will be achieved by the combination of receiver measures with GNSS authentication (Psiaki \& Humphreys, 2016).

Within the civil aviation domain, ICAO Standards and Recommended Practices (Annex 10, vol. 1, s.10) (International Civil Aviation Organisation, 2006) explicitly mention that interference protection measures are related to unintentional interference, and "not intended to specifically address intentional 
interference", as that caused by jammers or spoofers. However, intentional interference has recently been proposed for consideration (Enge, Walter, \& Shallberg, 2017).

The case of SBAS spoofing, which is the motivation of this work, is of a special importance within the aviation domain but also beyond: most GNSS receivers are nowadays SBAS-enabled, and just by altering the SBAS data, all the SBAS-augmented satellites can be spoofed, leading to a coherently forged position. Therefore, enabling a SBAS without authentication may constitute a single point of failure for many users.

The authentication of SBAS messages and codes was suggested for WAAS in (Scott, 2003). WAAS authentication schemes based on the Timed Efficient Stream Loss-Tolerant Authentication (TESLA) protocol, and digital signatures, are also proposed in (Lo \& Enge, 2010) and (Enge \& Walter, 2014), respectively, and both schemes have also been preliminarily analyzed for EGNOS (Chiara, et al., 2016). The European Commission launched the EAST (EGNOS Authentication Security Test-bed) project in 2016 with the purpose of assessing SBAS authentication schemes and their impact in SBAS performance. Its final results are presented here. This article complements the previous work on SBAS authentication in several ways: First, by presenting and trading off various implementation options. Then, by assessing how SBAS authentication affects accuracy, availability and integrity requirements, both qualitatively and quantitatively. Finally, the article presents the results of an exhaustive performance analysis for various authentication schemes, message sequences, and signal components. The analysis is based on real GNSS measurements, and realistic EGNOS scheduling algorithms and message sequencing constraints.

After this introduction, the article describes the main drivers for SBAS data authentication design, namely: the use of the In-Phase (I) or Quadrature (Q) channels, the use of the L1 and/or L5 frequencies, the latency, and the cryptographic parameters. Later it presents the performance impact of some authentication schemes applied to EGNOS, the European SBAS. The article finalizes with some conclusions and recommendations.

\section{SBAS Message Authentication SChEMES ANd Design Drivers}

Data authentication is achieved by delivering an authentication tag associated to a message or to a message sequence, i.e. a message authentication code (MAC), or a digital signature (DS). This tag is unambiguously determined by a cryptographic key and the message itself. If the received SBAS data is not the same as that transmitted by the SBAS system, the verification check will detect an inconsistency, with a probability that depends on the length of the authentication tag and the security parameters of the underlying cryptographic functions. The proposed definition of the tag used for data authentication schemes in this article, is:

$$
\operatorname{tag}=S(k e y,[M T|| \text { Data }|| T O M \mid \text { status }])
$$

where $S$ is the signing algorithm, key is the cryptographic key (either symmetric or private), $M T$ is the message type(s), $\|$ denotes concatenation, Data stands for the plaintext to be signed, TOW is the Time of Week (understood as a general time tag) and status is the status of the authentication function (e.g. 'Operational' or 'Test'). SBAS data authentication techniques analyzed in EAST are defined according to the following categories:

- Whether they are based on a digital signature or a MAC through the TESLA protocol (Perrig, Canetti, Tygar, \& Song, 2000).

- Whether they are inserted in the L1 legacy SBAS message or in the L5 Dual Frequency Multi Constellation (DFMC) message, modulated in the in-phase channel of the SBAS BPSK signal (hereinafter In-Phase), or inserted in the quadrature channel of either L1 or L5 (hereinafter In- 
Quadrature), currently unused, as the current standard (RTCA SC-159, 2006) only refers to one signal component.

\section{Generation and Verification of SBAS Message Authentication Data}

A detailed explanation of the cryptographic principles and operations of digital signatures and TESLA fall out of the scope of this paper and are available in (International Standards Organisation, 2009) and (Perrig, Canetti, Tygar, \& Song, 2000). This section presents an overview of how they are implemented, from cryptographic key generation to user verification. For simplicity, we start describing the case of digital signatures, where the basic principles are:

- The SBAS provider generates a public/private cryptographic key pair, and keeps the private key secret (key as per Eq. 1).

- The SBAS provider, or another trusted entity, publishes the public key so that receivers can incorporate it from factory.

- Once in operation, the receiver verifies the authenticity of the SBAS messages with the digital signature and the public key already in its possession.

For TESLA, the same principle is followed, except that the verification is performed through a MAC, associated to a key transmitted some seconds later, where the key is part of a one-way chain disclosed in reverse order with respect to its generation, whose final element, called root key, is authenticated by the public key. Therefore, for the TESLA authentication protocol, the user must:

- Have an independent and trusted timing reference, or loose time synchronization, whose maximal time error is lower than the delay between MAC and key disclosure;

- Receive a MAC $\left(M A C_{i}\right)$, and after the disclosure time, a key $\left(k_{i}\right)$.

- Verify the key's authenticity against a previous issued key $\left(k_{i-1}\right)$, or the chain root key $k_{0}$.

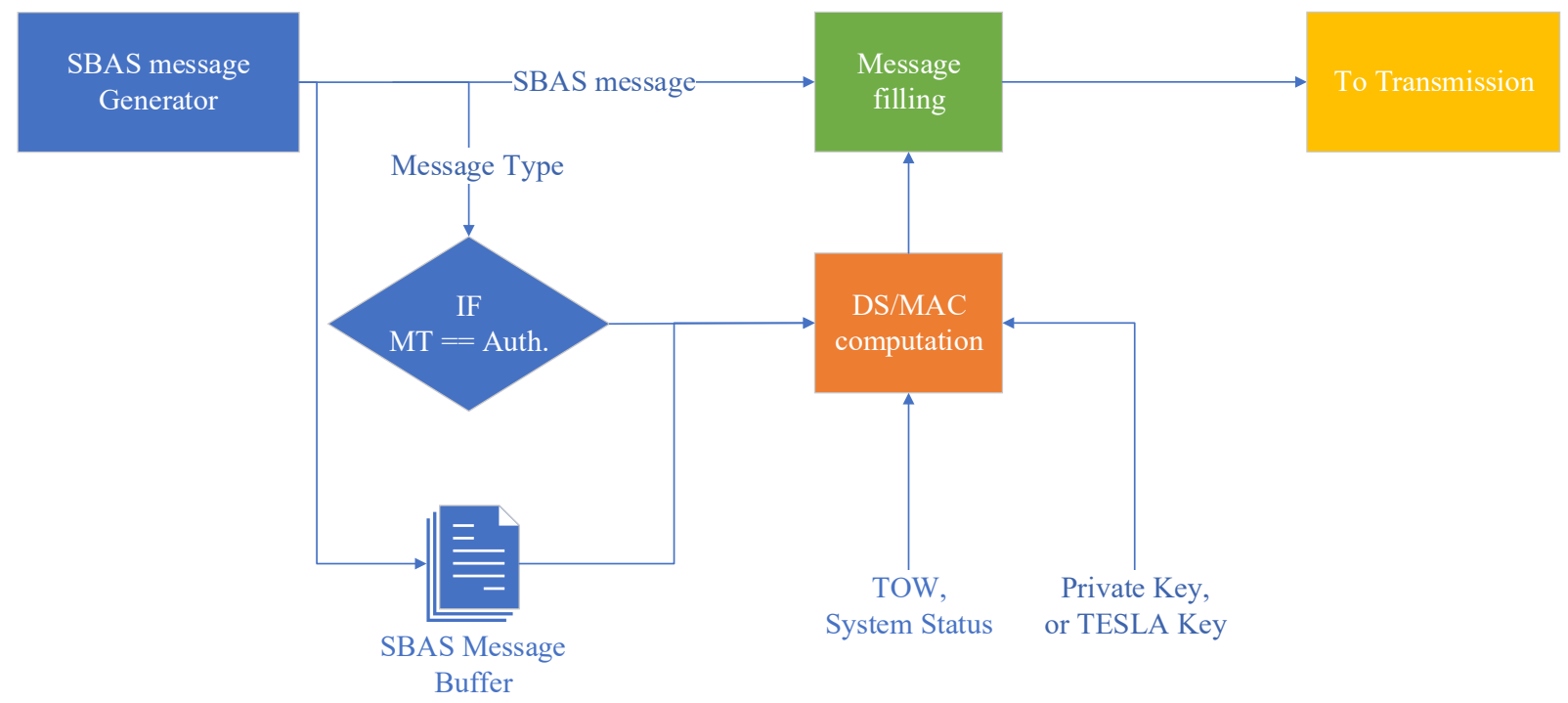

Figure 1 - SBAS message authentication scheme

Generally, SBAS data is fresh and expires rapidly, meaning that the data carried by each new message is generated just before it is broadcast, and its expected lifetime is generally limited to a few minutes at most, in contrast with the GNSS navigation data which can be valid for hours. Figure 1 sketches a basic solution to introduce SBAS authentication messages. An SBAS Message Generator generates a message every second, and an SBAS Message Buffer stores the messages. If the system scheduler assigns an authentication Message Type (MT) for transmission, the DS/MAC computation module authenticates the data stored in the Message Buffer, the authentication message is transmitted, and the buffer is emptied. This scheme is simplistic in that it assumes that the authentication data fits in a single message, and there is no redundancy or recovery mechanism, 
but it is illustrative of the process. In this scheme, all the public cryptographic information is assumed to be static and in the possession of the receiver. In an operational scheme, a part of the Authentication Message will be reserved to public key management, including key renewal when necessary, or revocation.

What follows is a summary of the main aspects driving the SBAS message authentication design, namely:

- Use of the I or Q channels, and messages to protect by each authentication message.

- Use of L1 or L5 message sequence.

- Cryptographic security parameters and public key management.

- Time Between Authentications and authentication latency.

\section{I or Q Channel and Messages to Authenticate}

At the moment, SBAS standards, both for L1 (RTCA SC-159, 2006) and L5 (SBAS Interoperability Working Group Issue 1 Revision 3 April 2016, April 2016), which is currently in draft mode, only use the I signal component, leaving the Q component unused. Therefore, one of our main design choices is whether the authentication information is inserted in the already existing SBAS message sequence, or rather it is inserted in the Q channel. If in the I-channel, the scheme inserts additional messages which authenticate previous SBAS messages (note that the article does not consider SBAS "cross-authenticating" GNSS data). These may be limited to the not-yet-authenticated ones, or include a combination of authenticated and not-yet-authenticated messages, in a redundant fashion, to provide some message recovery capabilities. SBAS systems generate a message every second and broadcast it immediately. Thus, only present or past messages can be authenticated, as shown in Figure 1, but not messages to be broadcast in the future. I-channel schemes inevitably impact the SBAS scheduling algorithm, as even if they utilize existing unused bandwidth, they increase the update rate of other SBAS messages.

In-Quadrature (Q) authentication schemes do not consume any bandwidth in the I-channel, implying no delay in the transmission of SBAS data and can provide lower authentication latency than I-channel schemes. However, the signal power available for transmission must be shared between the I and Q components. Figure 2 depicts I and Q options, based on a standard SBAS 250bit message composed by a preamble ( 8 bits), a message type identifier ( 6 bits), the data field (212 bits in L1) and a CRC (24 bits).

a) SBAS Data and Authentication Data on I channel

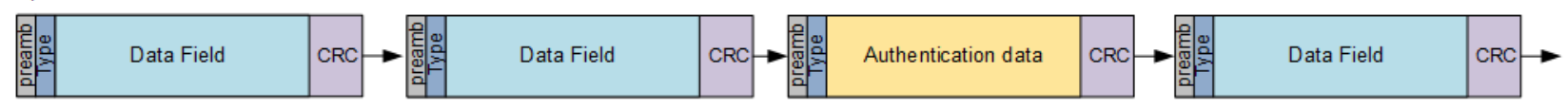

b) SBAS Data on I channel and Authentication Data on Q channel

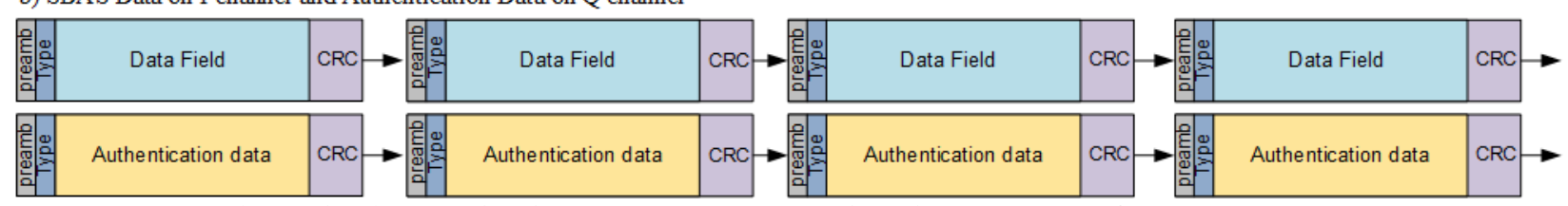

Figure 2 - Authentication data broadcast options in I (top) and Q (bottom) channels.

\section{L1 or L5}

If the I channel is used, authentication must be inserted in the legacy L1 sequence, in the L5 DFMC sequence, or in both sequences. The available bandwidth for authentication depends on several factors, as the number of augmented satellites/constellations, the ionosphere monitoring information (transmitted in L1 but not in L5), the service area, which determines the amount of L1 ionospheric monitoring information, or the SBAS message scheduler rules. Table 1 summarizes the bandwidth already in use in some representative cases based on EGNOS. The first row provides a reference case for the current use of L1 (single constellation), while the second row provides a similar 
reference for L5 (multi-constellation). All cases show that there is bandwidth available for authentication.

\begin{tabular}{|c|c|c|c|c|c|}
\hline Signal & $\mathbf{N}^{0}$ of GPS/GAL/GEO SV & MT 28 & $\begin{array}{l}\mathbf{N}^{\mathbf{o}} \text { of } \mathbf{S V} \\
\text { Monitored }\end{array}$ & Number of IGPs & $\begin{array}{l}\text { Bandwidth } \\
\text { Usage }\end{array}$ \\
\hline L1 & $31 / 0 / 3$ & Yes & 15 & ECAC (Europe) & $70 \%$ \\
\hline L5 & $31 / 0 / 3$ & Yes & 15 & No IONO (dual-freq) & $60 \%$ \\
\hline L1 & $24 / 26 / 1$ & Yes & 25 & ECAC (Europe) & $90 \%$ \\
\hline L5 & $24 / 26 / 1$ & Yes & 25 & No IONO (dual-freq) & $80 \%$ \\
\hline
\end{tabular}

Table 1 - Typical Bandwidth Usage in SBAS L1 and L5 without authentication

\section{Cryptographic Parameters and Public Key Management}

In order to allow public verification while retaining private credentials for signing, and to avoid distributing the signing credentials to all devices that need to perform verification, asymmetric schemes have been selected. The two schemes retained are based on EC-Schnorr digital signatures (International Standards Organisation, 2009) and TESLA (Perrig, Canetti, Tygar, \& Song, 2000). Both authentication schemes have been widely studied and proposed for GNSS as well as other radionavigation services (Lo \& Enge, 2010) (Margaria, et al., 2017) (Becker, et al., 2009) (Wullems, Pozzobon, \& Kubik, 2005) (Walker, et al., 2015). Other schemes based on different implementations and cryptographic protocols may be possible, but these schemes are representative enough for the feasibility and performance assessment.

The cryptographic parameters depend on the target bit security level (SL). For example, 128 bits of security $(\mathrm{SL}=128)$ imply that an exhaustive key search, or brute force attack, would require around $2^{128}$ trials to break the scheme. In symmetric cryptography, the security level is generally equivalent to the size of the key. In asymmetric cryptography, keys and digital signatures are generally longer in bits than the target security level, as shown later for EC-Schnorr. A target security level of 128 bits has been chosen for the analysis, based on standard recommendations from the cryptographic community (ENISA, 2013), and considering the expected life of the SBAS service. However, 80-bit security schemes may be sufficient in some cases because the SBAS and GNSS information is only relevant for a period between seconds (e.g. SBAS data), hours (e.g. ephemeris data) or months (e.g. TESLA key chain), allowing equally short cover times, achievable with shorter keys. Table 2 shows a qualitative comparison of the retained schemes, from the point of view of cryptographic security, bandwidth, availability based on AER (Authentication Error Rate), key management, and receiver requirements. We define AER as follows:

$$
A E R=\left(1-P E R_{m}\right)^{M}\left(1-P E R_{a}\right)^{A}
$$

Where $M$ is the number of SBAS messages authenticated, and $A$ is the number of messages carrying authentication information, and PER (Page Error Rate) represents the message error rate of the data message $\left(P E R_{m}\right)$ and the authentication message $\left(P E R_{a}\right)$ channels. When both data and authentication messages are transmitted both in the same (I) channel, $A E R=(1-P E R)^{M+A}$. While TESLA is cryptographically secure, it requires loose time synchronization between the transmitter and the receiver independent from the signal in space. The time synchronization required depends on the time between the reception of the MAC and its related key. Current designs can accommodate seconds to minutes of synchronization error. Allowing a higher synchronization error facilitates the implementation of the TESLA protocol in the receiver, but at the expense of adding latency to the authentication verification, which impact receiver performance. See for example (Fernández-Hernández, y otros, 2016) (fig. 7), where MACkey delays proposed are between a minimum of 10 seconds, and a maximum of beyond two minutes, in order to cope with different receiver implementations. If needed, avionics receivers may re-synchronize with GNSS when in a controlled environment, such as an airport prior to takeoff. If future receivers are upgraded with resilience measures, of which SBAS authentication may be one 
of them, independent time synchronization may be required anyway to protect ranging measurements.

\begin{tabular}{|c|c|c|c|c|c|}
\hline & Security & Bandwidth(*) & AER & $\begin{array}{c}\text { Key } \\
\text { Management }\end{array}$ & $\begin{array}{c}\text { Receiver } \\
\text { Requirements }\end{array}$ \\
\hline $\begin{array}{l}\text { EC- } \\
\text { Schnorr } \\
\text { (DS) }\end{array}$ & $\begin{array}{l}\text { Based on EC, Security } \\
\text { proof in Random } \\
\text { Oracle model }\end{array}$ & $\begin{array}{c}4 \times \text { SL for one } \\
\text { authentication: } \\
\sim 320 \text { bits }(\mathrm{SL}=80) \text { to } 512 \\
\text { bits }(\mathrm{SL}=128)\end{array}$ & $\begin{array}{l}\text { Depends on } \\
\text { digital } \\
\text { signature } \\
\text { reception } \\
\end{array}$ & $\begin{array}{c}\text { Few-bit low- } \\
\text { latency OTAR } \\
\text { message }\end{array}$ & $\begin{array}{c}\text { Public key (few-byte) } \\
\text { storage capacity } \\
\text { Low CPU }\end{array}$ \\
\hline TESLA & $\begin{array}{c}\text { Based on MAC } \\
\text { function (e.g. HMAC } \\
\text { (National Institute } \\
\text { of Standards and } \\
\text { Technology, FIPS } \\
\text { PUB 198-1: The } \\
\text { Keyed-Hash } \\
\text { Message } \\
\text { Authentication } \\
\text { Code (HMAC), } \\
\text { 2008)) and one-way } \\
\text { function (e.g. SHA- } \\
\text { 256 (National } \\
\text { Institute of } \\
\text { Standards and } \\
\text { Technology, FIPS } \\
\text { PUB 180-4: Secure } \\
\text { Hash Standard } \\
\text { (SHS), 2012)). } \\
\text { Security proof } \\
\text { available in (Perrig, } \\
\text { Canetti, Tygar, \& } \\
\text { Song, 2000). }\end{array}$ & $\begin{array}{c}\text { Key: } \sim \mathrm{SL}+\sim 15 \text { bits } \\
\text { MAC: } \sim 30 \text { bits } \\
\text { Total for one authentication: } \\
\sim 125 \text { bits }(\mathrm{SL}=80 \text { ) to } 173 \\
\text { bits }(\mathrm{SL}=128)\end{array}$ & $\begin{array}{l}\text { Depends on } \\
\text { MAC + key } \\
\text { reception. } \\
\text { Tolerant to } \\
\text { key losses }\end{array}$ & $\begin{array}{c}\text { Few-bit low- } \\
\text { latency OTAR } \\
\text { message }\end{array}$ & \begin{tabular}{|c} 
Public key (few-byte) \\
storage capacity \\
Low CPU \\
Loose time \\
synchronization
\end{tabular} \\
\hline
\end{tabular}

Table 2 - Qualitative comparison between data authentication schemes; (*) excluding public key management and general status overhead (AER: Authentication Error Rate; EC: Elliptic Curve; SL: Security Level; OTAR: Over-The-Air Rekeying)

As shown in Table 2, both EC-Schnorr and TESLA schemes need a few bits for over-the-air rekeying (OTAR). OTAR bandwidth is similar in both cases and can be accommodated in the extra bits of the SBAS authentication message with no or little impact in the scheme performance.

Discussing the security of authentication mechanisms, we distinguish two types of attacks: keyrecovery attacks and (selective) forgery attacks. In the former type, the attacker recovers the longterm secret key and can use it to compute the digital signature or the tag for any message. In the latter type, the attacker can produce the digital signature or the tag for one or a small set of messages. In MAC-based schemes like TESLA, key-recovery attacks start from observed messagetag pairs and look for keys that produce matching tags for the observed messages. The attacks can sometimes be accelerated by means of precomputed tables.

EC-Schnorr requires a digital signature length of four times the target security level (SL), or 512 bits for 128 bits of security ( $\mathrm{SL}=128$ ). For TESLA, SL is generally equivalent to the TESLA key length, but the scheme designers propose to take an extra margin of 15 bits to compensate the probability of finding second preimages within the TESLA key chain. A second preimage of a TESLA key $K_{i}$ is a key $K_{i+1}^{\prime}$ different from $K_{i+1}$ used to generate $K_{i}$ through the one way function $\mathrm{F}$, such that $\mathrm{F}\left(\mathrm{K}_{\mathrm{i}+1}^{\prime}\right)=\mathrm{F}\left(\mathrm{K}_{\mathrm{i}+1}\right)=\mathrm{K}_{\mathrm{i}}$. These additional bits in the TESLA key constitute a security 
margin aimed at making the scheme more robust to second preimage attacks. A detailed description of the TESLA key derivation is reported in (Caparra, Sturaro, Laurenti, \& Wullems, 2016), which considers that hardware with hashing rate as high as $5 \cdot 10^{13}$ hash/s are practically available on the market, and (Walker, y otros, 2015), which proposes a time-dependent one-way function.

If TESLA is used with a secure MAC function, like e.g. HMAC (Keyed-Hash MAC) (National Institute of Standards and Technology, FIPS PUB 198-1: The Keyed-Hash Message Authentication Code (HMAC), 2008), the MAC tag cannot be distinguished from a random bit sequence. In order to forge a tag without doing a full key recovery, the attacker can only make a random guess for the tag and hope that it will match with the right tag for the message that he forged. The probability of success of this attack is small, and decreases exponentially with the tag length. Furthermore, the attacker does not obtain any advantage from observing tags for previous messages and faces the same small probability of success for every new message that he wants to forge. For example, if the tag were $\mathrm{SL}=128$ bits, it would be as low as $2^{-128}$, or $2.9 \cdot 10^{-39}$ for each message.

Therefore, the TESLA tag can be smaller than SL. An attack can lead to an integrity failure, whose probability, according to the integrity requirement, must be below $2 \cdot 10^{-7}$ per approach (International Civil Aviation Organisation, 2006). We therefore assume that a successful attack with a probability below $2 \cdot 10^{-9}$ is low enough to have no impact in the SBAS safety case. A 29-bit tag would yield a success probability of $2^{-29}$, or $1.86 \cdot 10^{-9}$ and therefore would be enough. One could argue that an attacker can maximize his chances by making several attempts over an approach procedure. However, as soon as an authentication event fails, an alert can be triggered, preventing the success of the next attempts.

For comparison purposes, a 24-bit message CRC is defined by SBAS MOPS (RTCA SC-159, 2006) to provide "protection against burst as well as random errors with a probability of undetected error $\leq 2^{-24}=5.96 \cdot 10-8$ ", which yields a negligible integrity failure probability when conditioned to the expected message error rate of $10^{-3}$ for SBAS nominal users, and the fact that two SBAS GEO links are required to maintain the continuity requirement.

Therefore, the 212-bit data payload of a single L1 SBAS message can easily accommodate one 143bit key $(128+15)$ and a long-enough tag (e.g. 30 bits, as proposed), leaving some free space (39 bits in this case) for OTAR, status reporting or other purposes. Even if the protocol had to protect against an attacker having multiple opportunities to guess, the format would not be significantly altered, as adding a single bit to the tag would reduce the success probability by half, and so on. We must also note that we are assuming an attack probability of 1 , which is a pessimistic assumption. For EC-Schnorr, we will assume a 512-bit signature accommodated in three SBAS messages (636 bits), leaving 124 bits, 41 or 42 bits per message, for OTAR and other purposes.

Regarding the public key management, once the first public key or keys are loaded from the factory, the proposed schemes require no operator maintenance during the GNSS receiver lifetime, as the next public keys will be updated through the SIS. This can be done through low-latency, low-bitrate OTAR bits transmitted in the spare bits of the authentication message. However, in case of public key compromise, rekeying through a non-SIS channel, as e.g. the flight management system database in case of avionics receivers, may be required. A detailed explanation of the public key management schemes is beyond the scope of this article, but some examples of low-bandwidth schemes for GNSS are proposed in (Kerns, Wessons, \& Humphreys, 2014) or (Caparra, Ceccatto, Sturaro, \& Laurenti, 2017).

\section{Time Between Authentications (TBA) and Authentication Latency}

TBA, understood as the time between authentication verification events, is a relevant design parameter where robustness and performance degradation are traded-off. An authentication 
message transmitted frequently, e.g. every few seconds (low TBA), consumes significant bandwidth, potentially degrading performance; on the other hand, an authentication message transmitted infrequently, e.g. every few minutes (high TBA) increases the AER and forces the receiver to coast during a longer time using non-authenticated information.

Authentication latency, understood as the maximum time between reception of a message and its authentication, is also a relevant parameter given that, unlike GNSS ephemerides, SBAS messages are continuously changing. Authentication latency has a direct impact in the Time To Alert (TTA) associated to the integrity requirement, which is discussed later in the performance results section. We assume that a desirable authentication latency should be 6 seconds or less, as 6 seconds is the time for an SBAS to detect and report an event affecting the service integrity. Latency and TBA are interrelated and its relationship depends on the scheme, as shown in Figure 3.

Considering the channel flexibility (I/Q) and the authentication schemes (TESLA/EC-Schnorr), four implementations were developed by Qascom and Padua University and presented in Figure 3. Figure 3 also shows that the authentication messages include some extra bits, reserved for the authentication status, the DS page, and some key management overhead for public key distribution. For simplicity the same bitrate in the I and Q signal components has been considered. Different bitrates for the $\mathrm{Q}$ channel are analysed later in this article. The following paragraphs present the pros and cons of the different schemes, as per Figure 3.

The first scheme depicted is the In-Phase using EC-Schnorr digital signatures, or ECSch-I. In this scheme, $\mathrm{B}_{\mathrm{i}}, \mathrm{B}_{\mathrm{i}+1}$, etc., are blocks of SBAS messages to be authenticated. "Auth.Msg" indicates one authentication message, which mainly consists of a digital signature $(D S)$. As signatures could not fit in a single message, the message scheduler used has to schedule messages in between, in order to maintain their update intervals. These spread blocks of messages (e.g. composed by $\mathrm{B}_{\mathrm{i}+1}, \mathrm{~B}_{\mathrm{i}+2}$ and $\mathrm{B}_{\mathrm{i}+3}$ in Figure 3) are aggregated and authenticated in the next signature $\mathrm{DS}_{2}$. This has an impact in the authentication latency (time between the first message of $\mathrm{B}_{\mathrm{i}+1}$ is received and authenticated), and the TBA (time between $\mathrm{DS}_{1}(3 / 3)$ and $\mathrm{DS}_{2}(3 / 3)$ are received).

The second scheme, ECSch-Q, implements EC-Schnorr signatures in the Q channel. A 50-50\% power sharing and the same bitrate in both I and Q components is considered. In the example, every three messages, a full signature is transmitted, e.g. $\mathrm{DS}_{2}$, authenticating three messages $\mathrm{M}_{\mathrm{i}+2}, \mathrm{M}_{\mathrm{i}+3}$ and $\mathrm{M}_{\mathrm{i}+4}$, the latter transmitted in parallel with the first message carrying $\mathrm{DS}_{2}$. TBA is three seconds, and latency four seconds: the time between the earliest message $\mathrm{M}_{\mathrm{i}+2}$ is received, and it is authenticated after full reception of $\mathrm{DS}_{2}$.

The third scheme, TESLA-I, implements the TESLA protocol in the In-Phase channel. We assume that one authentication message carries at least a MAC and a key associated to the previous MAC. TBA is the time between two authentication messages, and the latency is higher, as an authentication event requires the MAC from the previous authentication message.

In the fourth scheme, TESLA-Q, the TESLA protocol is implemented in the Q channel. In this case, an authentication message can be transmitted every second $(\mathrm{TBA}=1 \mathrm{~s})$, as we assume the same bitrate of $250 \mathrm{bps}$ in both components. The latency is also 1s, i.e. as the receiver only needs to wait for the key in the next second to authenticate the previous message. Note that, in both TESLA-I and TESLA-Q cases, a loose time synchronization requirement of 1 second is assumed. Even if the time between the message containing the MAC and the message containing the corresponding key is greater in the TESLA-I case, a higher time uncertainty may allow data collision attacks, in which the attacker gets the key and modifies the last part of the data with a different dataset that still produces the same tag. 

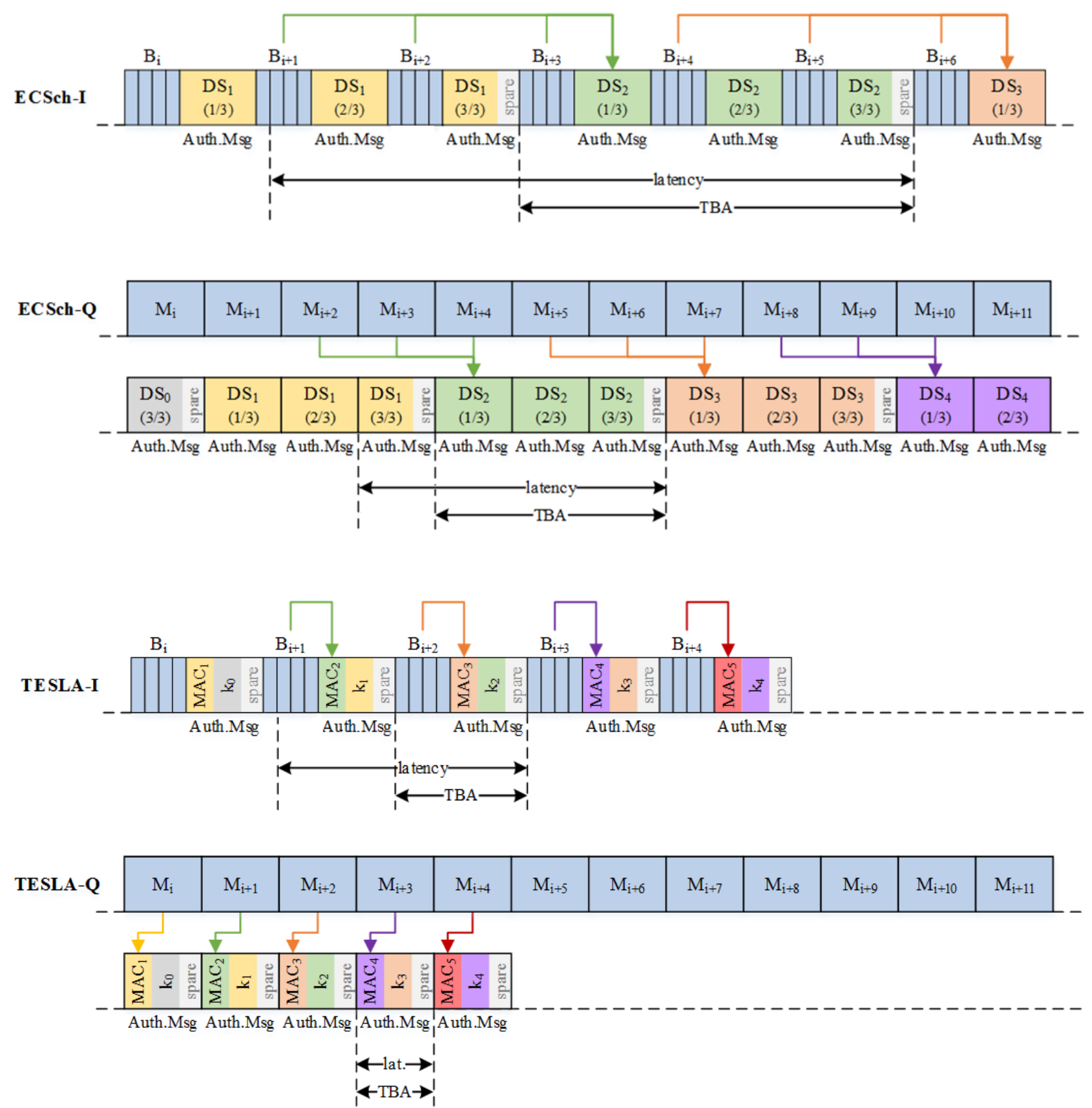

Figure 3 - Simplified scheme of the implementations of SBAS message authentication, using EC-Schnorr and TESLA solutions, applied to the In-Phase and Quadrature configurations. B indicates a contiguous set of SBAS messages (block); $M$ indicates one single SBAS message. The EC-Schnorr solution presented makes use of three Auth. messages to deliver one single signature; the TESLA solution makes use of one Auth. message to deliver a MAC and a key (the two do not correspond to the same verification). All the auth. messages also include room for spare bits, typically reserved to OTAR and status reporting.

\section{Performance Results}

This section summarizes the performance results of the proposed sample authentication schemes, when introduced in the EGNOS signal and sequence. First, the authentication solutions are assessed through three authentication Key Performance Indicators (KPIs): AER, TBA, and latency. The adequacy of these indicators as a proxy for the user performance is further justified in (FernándezHernández I. , 2014). Then, the impact of the authentication schemes in EGNOS performance is evaluated. The analysis does not consider performance under attacks, but only under nominal 
conditions. However, the data spoofing threat is considered theoretically in the analysis of the integrity requirement.

The performance results were obtained with the simulator of EAST, the project under which these analyses have been performed. In the simulator, a message sequence is generated with real EGNOS sequences from EDAS (EGNOS Data Access Service) (European GNSS Agency, 2014) and GNSS data from RINEX files. This message sequence and data is altered to accommodate the authentication messages by a modified EGNOS message scheduler algorithm from GMV. It is then passed onto a physical layer where the symbols encoding the data are generated. The channel can be modelled as Additive White Gaussian Noise (AWGN) or Land Mobile Satellite (LMS) (International Telecommunication Union, 2015). Finally, the Application/Performance Layer analyzes the SBAS user and authentication performance.

\section{Authentication Performance and I vs. Q channel Apportionment}

Table 3 presents a performance summary of the different data authentication solutions. We assumed that three SBAS 212-bit messages are required for an EC-Schnorr authentication message (636 bits) and one single SBAS 212-bit message for TESLA, both allowing 128 equivalent security bits. The maximum update interval (or maximum TBA) was configured to 6 and 18 seconds for TESLA and EC-Schnorr respectively, leading to the same bandwidth consumption. The message scheduler inserted the messages more often though, leading to average TBAs in the order of 5 and 13 seconds respectively.

Table 3 first shows the minimum $\mathrm{C} / \mathrm{N}_{0}$, including the signal power of both $\mathrm{I} / \mathrm{Q}$ when $\mathrm{Q}$ is used, required for an AER of $1 \%$. In these conditions, the average TBA is also reported. While a 1\% AER may be too high for aviation applications, its characterization may be useful for other SBAS users and for comparison purposes. For example, for L1-I EC-Schnorr, a 1\% AER is achieved with a $\mathrm{C} / \mathrm{N}_{0}$ of $28.5 \mathrm{dBHz}$. In these conditions, the average TBA is 13.52 seconds, taking into account that the scheduler inserts the signatures more often than every 18 seconds, but that $1 \%$ of the authentications will fail. The maximum latency range between 20-29 seconds represents the difference between inserting the three DS messages as close as possible (20-second latency), and inserting one every 6 seconds, which is a worst, non-optimized case. The 29 -second latency can be explained by Figure 3, ESch-I case (top), if data/signature messages are transmitted at a ratio of 5/1, instead of 4/1. It implies that the receiver has to wait for 20-29 seconds between a message is received, and it is authenticated. This means it has to coast for 14 to 23 seconds beyond the 6second TTA with an unauthenticated message. While TESLA schemes in the I channel allow more frequent authentications than EC-Schnorr, the latency of the proposed schemes do not fulfil the 6second TTA due to the need to receive two messages, one with the MAC and one with the key.

The 6-second TTA is only fulfilled in the Q-channel schemes. However, as expected, a I-Q 50/50\% apportionment of the existing power highly degrades performance, or would require a much higher radiated power, which is not considered as a realistic option. A power apportionment of $75 \% / 25 \%$ for I/Q channels leads to a lower degradation of the I channel power relative to $100 \%$ power on the I channel, in the order of $1 \mathrm{~dB}$, while still within the 6-second TTA requirement.

\begin{tabular}{|c|c|c|c|c|c|}
\hline \multicolumn{2}{|c|}{$\begin{array}{c}\text { C/Nemes } \\
(\text { AER 1\%) }\end{array}$} & $\begin{array}{c}\text { Avg. TBA } \\
\text { (AER 1\%) }\end{array}$ & Max. TBA & $\begin{array}{c}\text { Max. } \\
\text { Latency }\end{array}$ & $\begin{array}{c}\text { SBAS } \\
\text { messages } \\
\text { per Auth. }\end{array}$ \\
\hline L5-I ECSch & $28.5 \mathrm{dBHz}$ & $13.52 \mathrm{~s}$ & $18 \mathrm{~s}$ & $20-29 \mathrm{~s}$ & 3 \\
\hline L5-I TESLA & $28.3 \mathrm{dBHz}$ & $5.9 \mathrm{~s}$ & $6 \mathrm{~s}$ & $11 \mathrm{~s}$ & 2 \\
\hline L1-I ECSch & $28.5 \mathrm{dBHz}$ & $12.89 \mathrm{~s}$ & $18 \mathrm{~s}$ & $20-29 \mathrm{~s}$ & 3 \\
\hline L1-I TESLA & $28.3 \mathrm{dBHz}$ & $4.89 \mathrm{~s}$ & $6 \mathrm{~s}$ & $11 \mathrm{~s}$ & 2 \\
\hline
\end{tabular}




\begin{tabular}{|c|c|c|c|c|c|}
\hline Q ECSch 50/50\% & $31.3 \mathrm{dBHz}$ & $3.03 \mathrm{~s}$ & $3 \mathrm{~s}$ & $4 \mathrm{~s}$ & 3 \\
\hline Q TESLA 50/50\% & $31 \mathrm{dBHz}$ & $1.01 \mathrm{~s}$ & $1 \mathrm{~s}$ & $1 \mathrm{~s}$ & 1 \\
\hline Q ECSch 75/25\% & $29.1 \mathrm{dBHz}$ & $5.05 \mathrm{~s}$ & $5 \mathrm{~s}$ & $8 \mathrm{~s}$ & $\begin{array}{c}5 \\
\text { (code rate } 1 / 4, \\
\text { see Table 4) }\end{array}$ \\
\hline Q TESLA 75/25\% & $29.3 \mathrm{dBHz}$ & $2.02 \mathrm{~s}$ & $2 \mathrm{~s}$ & $4 \mathrm{~s}$ & $\begin{array}{c}2 \\
\text { (code rate } 1 / 4, \\
\text { see Table 4) }\end{array}$ \\
\hline
\end{tabular}

Table 3 - Authentication KPI Summary

In order to further understand and minimize the degradation from using the Q channel, some basic improvements are proposed. They are based on the removal of redundant information, and different coding rates and power ratios between I and Q components.

Firstly, the encoding of the authentication message in the Q channel does not require the preamble ( 8 bits) and the MT identifier (6 bits). This assumes that the receiver can synchronize the SBAS data with the I component, which carries this information. Also, the 24-bit CRC per message can be replaced by a lighter redundancy check. These changes increase the information bit rate on the Q channel. Secondly, in order to maintain the overall transmitter and receiver complexity, three convolutional code schemes are proposed as examples: the first with the current SBAS L1 coding rate $R c=1 / 2$, and two more with $R c=1 / 3$ and $R c=1 / 4$. Some of these options decrease the Qchannel bit rate, requiring more time to transmit the authentication information, as shown in the last column of Table 3. The two candidates are derived from (Lin \& Costello, 2004) and their properties are described in Table 4, where the polynomial $\mathrm{G}(\cdot)$ associated with the coding scheme is expressed in octal form. The two candidates are here reported as an example to assess the impact of a lower bit rate on the authentication solutions, and they are not specifically tailored to the SBAS service.

\begin{tabular}{|c|c|c|c|c|c|c|}
\hline Code rate & \multicolumn{1}{c|}{$\begin{array}{c}\text { Constraint } \\
\text { length }\end{array}$} & $\mathbf{G}(\mathbf{0})$ & $\mathbf{G ( 1 )}$ & $\mathbf{G ( 2 )}$ & $\mathbf{G ( 3 )}$ & $\begin{array}{c}\text { Q-channel } \\
\text { Bit-rate }\end{array}$ \\
\hline $\mathbf{1} / \mathbf{2}$ & 7 & 171 & 133 & & & $\mathbf{2 5 0} \mathbf{b i t} / \mathbf{s}$ \\
\hline $\mathbf{1} / \mathbf{3}$ & 9 & 575 & 623 & 727 & & $\mathbf{1 6 6} \mathbf{b i t} / \mathbf{s}$ \\
\hline $\mathbf{1} / \mathbf{4}$ & 9 & 533 & 575 & 647 & 711 & $\mathbf{1 2 5} \mathbf{b i t} / \mathbf{s}$ \\
\hline
\end{tabular}

Table 4 - Convolutional coding schemes used in the SBAS Q-channel analysis

Complementarily to the coding rates, different I vs. Q power apportionments than $50-50 \%$ have been studied. In particular $75 \%(\mathrm{I}) / 25 \%(\mathrm{Q})$, and $80 \%(\mathrm{I}) / 20 \%(\mathrm{Q})$, leading to an I-channel power loss of only $1.25 \mathrm{~dB}$ and $0.97 \mathrm{~dB}$, respectively, assuming the currently radiated power is maintained. 


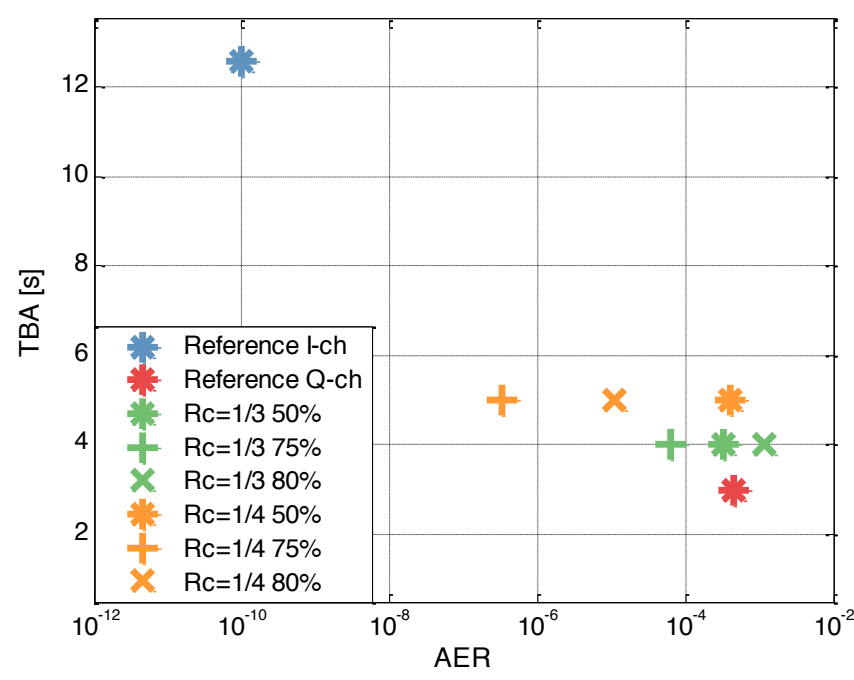

Figure 4 - EC-Schnorr AER vs TBA for all power split and code rate solutions examined at $32 \mathrm{dBHz}, \mathrm{L1}$

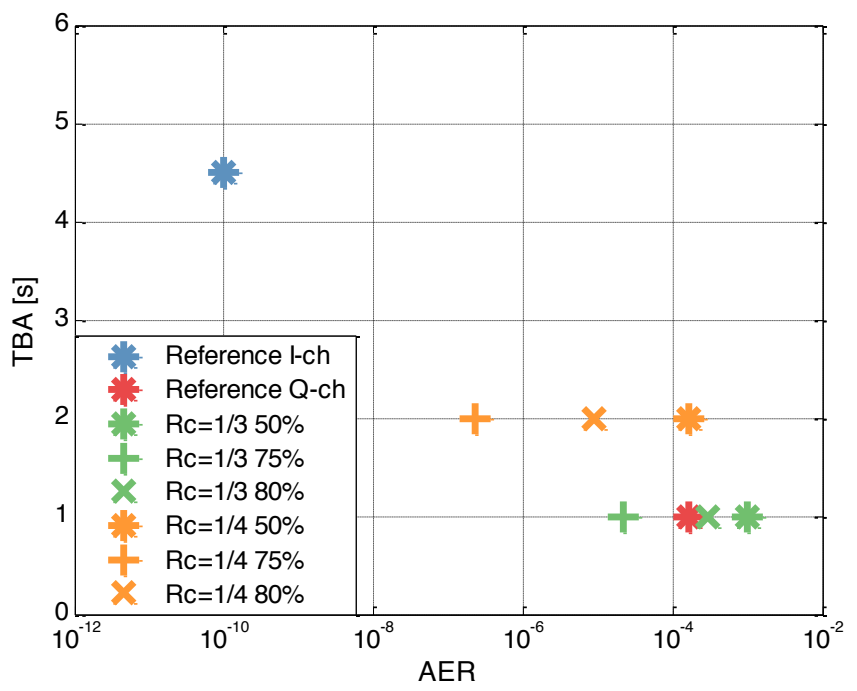

Figure 5 - TESLA AER vs TBA for all power split and code rate solutions examined at $32 \mathrm{dBHz}, \mathrm{L1}$

Figure 4 and Figure 5 depict the performance trend for each code rate and power split configuration, for EC-Schnorr and TESLA respectively. Each diagram presents the AER on the x-axis and the TBA on the $y$-axis, for different schemes, at a fixed $\mathrm{C} / \mathrm{N}_{0}$ of $32 \mathrm{dBHz}$. AER and TBA are computed numerically based on an analytical model of the SBAS L1 BER in an AWGN channel. The closer to the origin (lower TBA, and lower AER), the better the performance. The figures show that TBA (and, therefore, latency), is lower for TESLA than for EC-Schnorr, which is driven by the spacing of authentication events and the amount of failed authentications increasing TBA. The Q channel case (Reference Q-ch) with a 50/50\% power sharing and $R c=1 / 2$, and the I channel case (Reference I-ch), without using the Q channel, are provided as a reference. These optimizations show that, for example, halving the bitrate from $250 \mathrm{bps}$ to $125 \mathrm{bps}$ with a coding rate of 1/4, the EC-Schnorr scheme would achieve a 5-second TBA (and therefore be compatible with a 6-second TTA), with a high robustness at $32 \mathrm{dBHz}$, only at a decrease of around $1.25 \mathrm{~dB}$ of power $(75 / 25 \%)$ from the I channel. In these conditions, the TESLA scheme would obtain a TBA of 2 seconds. The approach also suggests that an even lower bitrate and power in the Q channel would still fulfill a 6-second TBA. Note that other schemes such as (Scott, 2003) use a much lower bitrate of 12.5 bps, or 75 bits every 6 seconds, which may be too low for the proposed cryptographic schemes.

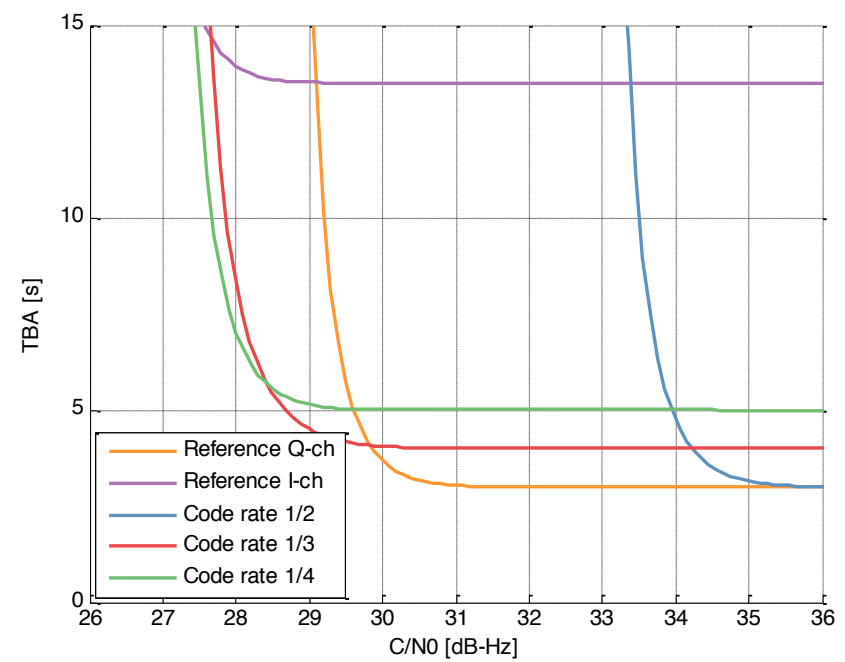

Figure 6 - EC-Schnorr TBA, 75\%/25\% power split, L1

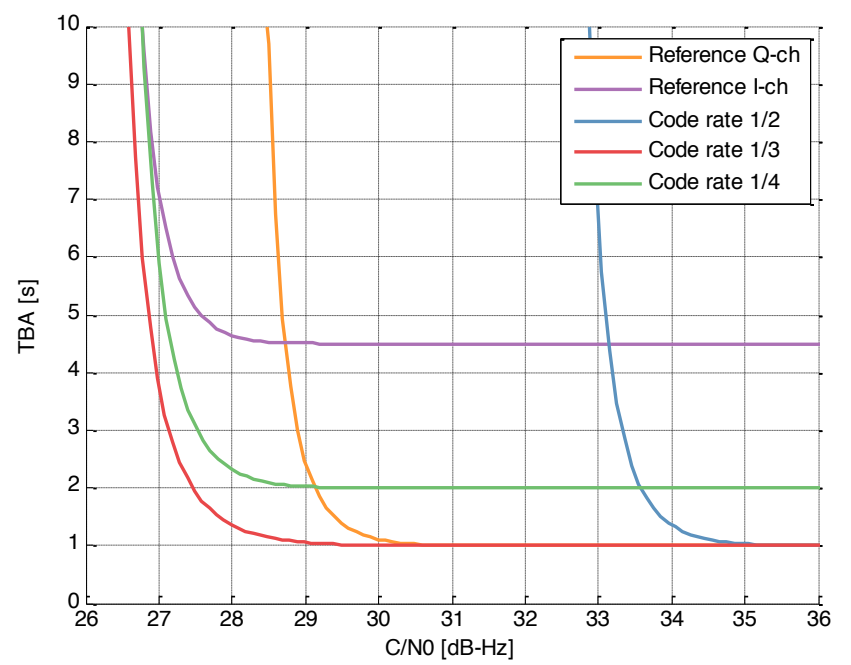

Figure 7 - TESLA TBA, 75\%/25\% power split, L1 
Figure 6 and Figure 7 show TBA at different $\mathrm{C} / \mathrm{N}_{0}$ values for each scheme and a power split fixed to $75 / 25 \%$, for EC-Schnorr (left) and TESLA (right). TBA is computed numerically based on an analytical model of the SBAS L1 BER in an AWGN channel. The figures show that, with a coding rate of $1 / 4$ (green), the $\mathrm{AER}$ vs. $\mathrm{C} / \mathrm{N}_{0}$ relationship is similar to that for the Reference I channel (i.e. all power allocated to the I channel), but with a much lower average TBA: from 13 seconds to 5 seconds for EC-Schnorr, and from 4.5 seconds to 2 seconds for TESLA.

\section{Impact of Authentication in SBAS Service Requirements}

This section presents the impact in SBAS performance of inserting authentication messages in the I channel of L1 (single constellation, 31 GPS satellites) and L5 (dual L1/L5 frequency, multi constellation, 24 GPS and 24 Galileo satellites). Before presenting the results, it is anticipated that the currently proposed authentication solutions are very bandwidth-intensive compared to, for example, the ones proposed in (Enge \& Walter, 2014), which relax TBA to the order of minutes, leading to a lower, or negligible, performance impact. This analysis assumes that the TTA is not waived for authentication, and therefore reduces the authentication latency as much as possible. Under these assumptions, Table 5 and Table 6 present the bandwidth used for L5 and L1 using ECSchnorr or TESLA, and assuming that the messages are transmitted at their maximum update intervals (except MT_D which is transmitted every $90 \mathrm{~s}$ and the maximum is $120 \mathrm{~s}$ ). The considered SBAS message scheduler was configured to provide at least three SBAS messages every 18 seconds (one EC-Schnorr authentication) or one SBAS message each 6 seconds (one TESLA authentication), leading to the same bandwidth usage. However, it was capable of allocating SBAS messages for authentication slightly more often (around four every 18 seconds), leading to a $22.2 \%$ bandwidth usage in both cases.

\begin{tabular}{|c|c|c|c|c|c|c|c|c|c|c|c|c|c|c|c|}
\hline Satc & MT1 & IT10 & MT12 & MT17 & MT18 & TT26 & MT27 & $\begin{array}{c}\text { MT- } \\
\text { AUTH }\end{array}$ & MT7 & MT9 & $\begin{array}{c}\text { MT } \\
\text { Fast,1sat }\end{array}$ & $\begin{array}{c}\text { MT } \\
\text { LongT }\end{array}$ & $\begin{array}{c}\text { MT- } \\
\text { UDRE }\end{array}$ & $\begin{array}{c}\text { Total BW } \\
\text { Auth }\end{array}$ & $\begin{array}{c}\text { Total } \\
\text { BW } \\
\text { No Auth }\end{array}$ \\
\hline 32 & $0.83 \%$ & $0.83 \%$ & $0.33 \%$ & $1 \%$ & $1.66 \%$ & $7.33 \%$ & $0.33 \%$ & $22.22 \%$ & $0.83 \%$ & $0.83 \%$ & $50 \%$ & $0 \%$ & $6.667 \%$ & $92.882 \%$ & $70.659 \%$ \\
\hline
\end{tabular}

Table 5 - Bandwidth computation for mono frequency (L1) single constellation scenarios

\begin{tabular}{|c|c|c|c|c|c|c|c|c|c|c|c|}
\hline $\begin{array}{l}\text { Satellites } \\
\text { in mask }\end{array}$ & $\begin{array}{c}\text { MT12- } \\
\text { ENHANCED }\end{array}$ & $\begin{array}{l}\text { MT- } \\
\text { AUTH }\end{array}$ & $\begin{array}{c}\text { MT6 } \\
\text { ENHANCED }\end{array}$ & MTB & MTC & MTD & MTE & MTF & MTG & $\begin{array}{c}\text { Total } \\
\text { BW } \\
\text { Auth }\end{array}$ & $\begin{array}{c}\text { Total } \\
\text { BW } \\
\text { No Auth }\end{array}$ \\
\hline 48 & $0.833 \%$ & $22.222 \%$ & $0.000 \%$ & $0.833 \%$ & $16.667 \%$ & $53.330 \%$ & $1.667 \%$ & $0.833 \%$ & $1.667 \%$ & $98.052 \%$ & $82.500 \%$ \\
\hline
\end{tabular}

Table 6 - Bandwidth computation scenarios for DFMC (L5) scenarios

Adding authentication to the SBAS message sequence affects performance due to the following effects:

- As additional bandwidth is used for authentication, the remaining messages are refreshed less often.

- For a successful authentication, several consecutive authentication and data messages have to be correctly received. When an authentication fails due to a data transmission error, the whole set of messages is discarded. This effect, whose impact is particularly visible in SBAS continuity requirement, can be mitigated by the addition of recovery messages as already proposed in (Enge \& Walter, 2014), but this feature has not been added yet to the proposed authentication schemes in the current analysis.

The results are based on a reference scenario lasting 24 hours, and composed of real SBAS and GNSS data, which is altered by the simulator to accommodate the authentication information. The scenario configurations reported here are:

- The reference scenario with and without authentication, assuming no reception failures $(\mathrm{BER}=$ $\mathrm{PER}=\mathrm{AER}=0 \%$ ). In this case, only authentication bandwidth impact is measured.

- The reference scenario with and without authentication, assuming a message loss rate, or Page Error Rate (PER), of $10^{-3}$, in line with SBAS MOPS (RTCA SC-159, 2006). This PER is 
equivalent to an AER of $0.3 \%$ and $0.5 \%$ for the L1/I TESLA and EC-Schnorr solutions, respectively, according to Eq. (2).

All SBAS KPIs are computed over a grid of users within the EGNOS service area. The SBAS KPIs analyzed characterize EGNOS performance in terms of accuracy, integrity, continuity and availability. They are assessed against requirements for LPV-200 (RTCA SC-159, 2006), Localizer Performance with Vertical guidance approach for 200-ft decision height, which is the target operation of SBAS, and are recalled in Table 7. The analysis focuses on the vertical domain for the Vertical Navigation System Error (V-NSE) and Vertical Protection Level (VPL), as the requirements are much more stringent than in the horizontal domain.

\begin{tabular}{|c|c|c|c|}
\hline Accuracy & Integrity & Continuity & Availability \\
\hline $\begin{array}{l}\text { V-NSE 95\%: } 4 \mathrm{~m} \\
\text { H-NSE 95\%: } 16 \mathrm{~m}\end{array}$ & $\begin{array}{c}\text { VAL: } 35 \mathrm{~m} \\
\text { HAL: } 40 \mathrm{~m} \\
\text { Integrity risk: } 2 \cdot 10^{-7} / 150 \mathrm{~s} \\
\text { TTA: } 6 \mathrm{~s}\end{array}$ & $\begin{array}{c}\text { Continuity risk: } \\
8 \cdot 10^{-6} / 15 \mathrm{~s}\end{array}$ & $\begin{array}{l}99.999 \% \text { to } 99 \% \text {, } \\
\text { depending on the } \\
\text { zone }\end{array}$ \\
\hline
\end{tabular}

Table 7 - LPV-200 requirements used as a reference for SBAS authentication performance

Accuracy is expressed as the Navigation System Error (NSE), or the difference between the real position of the aircraft and the position provided by the airborne equipment. Vertical Position Errors (VPE) at the $95^{\text {th }}$ percentile have been characterized.

Integrity, in the aviation domain (International Civil Aviation Organisation, 2006), is "a measure of the trust that can be placed in the correctness of the information supplied by the total system". It therefore has a different meaning than data integrity in the communication or cryptography domains, which assures that the data has not been altered. An SBAS integrity event may occur if for example a satellite clock runoff or a wrong broadcast ephemeris leads to an error in the SBAS receiver position, even if data integrity is maintained. Aviation integrity must also "include the ability of a system to provide timely and valid warnings to the user (alerts)" (International Civil Aviation Organisation, 2006). For LPV-200, this corresponds to the 6-second Time To Alert (TTA). This implies that SBAS has 6 seconds to detect and report an integrity threat, such as a wrong broadcast ephemeris, so an SBAS receiver must not be exposed to it for more than 6 seconds. If we treat spoofing events as integrity events, the SBAS authentication scheme could have a maximum latency of 6 seconds, as this means that the receiver could take 6 seconds between forged data is received, and the authentication verification detects the spoofing event. This latency is beyond the capability of the proposed I channel schemes, and would require using the Q channel.

A strict interpretation of authentication from the cryptography domain may imply that a message received without authentication is an incomplete message. This would force the receiver to wait until the authentication tag has arrived in order to process the data. While this interpretation is adequate for other cryptographic systems, it is hardly implementable in SBAS, as it requires to double or triple the bandwidth and does not allow fulfilment of the aviation TTA integrity requirement. On the other hand, an alternative approach could consider that the 6-second TTA is maintained for the data reception but not for the authentication of the data, which can be performed some time later. By allowing this approach, the receiver can coast without authentication for some minutes, as proposed in (Enge \& Walter, 2014), or some seconds, as proposed for the I channel schemes of this work. Integrity assurance in this case may be supported by combining authentication with receiver-based consistency indicators at signal, data or position level.

According to the SBAS integrity concept, an integrity event occurs when the vertical/horizontal position error (VPE/HPE) is above the vertical/horizontal protection level (VPL/HPL), which is a measure of the position uncertainty based on SBAS and receiver information. The case when VPE/HPE is also above the vertical/horizontal alert limit (VAL/HAL) is called "Hazardously 
Misleading Information" (HMI). While a 24-hour simulation with nominal data is not long enough to assess the integrity risk, it is not expected that the addition of authentication has any impact on it, because VPL/HPL will increase accordingly to the increase in VPE/HPE, ensuring safety even if the accuracy and availability performances are degraded. Therefore the performance analysis characterizes the impact of authentication focusing on the calculated VPL (RTCA SC-159, 2006), at the $99^{\text {th }}$ percentile.

Continuity is the probability that the specified system performance is maintained during the intended operation, which in our case is any 15 -second period, presuming that the system was available at the beginning of operation and predicted to be available during it. It is computed using a sliding window over time, and accounting for a continuity break within the window. A continuity break occurs when, at a given time, the system is available (Protection Levels (PLs) < Alert Limits (ALs)) and in the following time window of operation (15 seconds) there is at least one epoch in which the system becomes unavailable (PL $>$ AL or PL not available). The lack of continuity implies a risk because the operation must be aborted. The inverse of the continuity probability, the continuity risk, is shown in the figures.

Availability is the probability that the navigation service is available at the beginning of the planned operation. An SBAS is considered available when both VPL and HPL are below the corresponding ALs.

\section{SBAS Authentication Performance Results}

In order to assess the performance of SBAS authentication, several tests were run with the following setup:

- SBAS L1 scenarios used 24 hours of real EGNOS data from GEO PRN 120, starting on 15/07/2015 00:00:00 UTC. The SBAS messages and user error budgets followed the MOPS DO-229 standard (RTCA SC-159, 2006) and the GPS constellation available at the time and composed by 31 satellites was considered.

- SBAS L1/L5 Dual-Frequency scenarios used synthetic data, models and a configuration representative of the future version of EGNOS Dual-Frequency and Multi-Constellation (e.g. DFREs profile, degradation parameters etc.). The SBAS messages and the user error budgets followed the structure of the Dual-Frequency GPS/Galileo SBAS MOPS under development. A 24-SVs GPS constellation and 24-SVs Galileo constellation were considered.

Table 8 presents a summary of SBAS authentication performance impact in VPE, VPL, continuity and availability. It also presents the AER of each authentication solution, when proceeds. All the simulations were run for the ECAC service area $\left(-40^{\circ}\right.$ to $40^{\circ}$ of longitude, and 20 to $70^{\circ}$ of latitude). However, Table 8 presents the average VPE, VPL, continuity and availability of 28 equally distributed data points within a reduced area of $-12^{\circ}$ to $20^{\circ}$ of longitude, and $35^{\circ}$ to $60^{\circ}$ of latitude, covering ECAC land masses and therefore considered more representative of SBAS users.

In the second and third columns of Table 8, authentication performance impact is presented for a zero-PER. Column two presents the results without authentication, and column three with authentication. As both TESLA and EC-Schnorr schemes consume the same bandwidth, their performance in this case is equivalent. Columns three to five present the impact of EC-Schnorr and TESLA under PER $=10^{-3}$, with respect to the case without authentication.

\begin{tabular}{|c|c|c|c|c|c|}
\hline \multicolumn{4}{|c|}{ PER $=\mathbf{0}$} & \multicolumn{3}{c|}{ PER $=\mathbf{1 0}^{-3}$} \\
\hline & No Auth & EC-Sch/TESLA & No Auth & EC-Sch & TESLA \\
\hline VPE 95\% & $2.86 \mathrm{~m}$ & $2.90 \mathrm{~m}$ & $2.86 \mathrm{~m}$ & $2.94 \mathrm{~m}$ & $2.93 \mathrm{~m}$ \\
\hline
\end{tabular}




\begin{tabular}{|c|c|c|c|c|c|}
\hline VPL 99\% & $17.37 \mathrm{~m}$ & $17.57 \mathrm{~m}$ & $17.38 \mathrm{~m}$ & $17.93 \mathrm{~m}$ & $17.91 \mathrm{~m}$ \\
\hline $\begin{array}{c}\text { Continuity } \\
\text { Risk }\end{array}$ & $<8 \cdot 10^{-6}$ & $<8 \cdot 10^{-6}$ & $<8 \cdot 10^{-6}$ & $7.7 \cdot 10^{-3}\left({ }^{1}\right)$ & $5.9 \cdot 10^{-3}\left({ }^{1}\right)$ \\
\hline $\begin{array}{l}\text { Availability } \\
\text { (PL < AL) }\end{array}$ & $99.71 \%$ & $99.63 \%$ & $99.71 \%$ & $98.80 \%$ & $99.19 \%$ \\
\hline \multirow[t]{4}{*}{ AER } & $\mathrm{N} / \mathrm{A}$ & 0 & $\mathrm{~N} / \mathrm{A}$ & $0.5 \%$ & $0.3 \%$ \\
\hline & \multicolumn{5}{|c|}{ L5-I } \\
\hline & \multicolumn{2}{|c|}{$\mathrm{PER}=0$} & \multicolumn{3}{|c|}{ PER $=10^{-3}$} \\
\hline & No Auth & EC-Sch/TESLA & No Auth & EC-Sch & TESLA \\
\hline VPE 95\% & $1.66 \mathrm{~m}$ & $1.72 \mathrm{~m}$ & $1.66 \mathrm{~m}$ & $1.72 \mathrm{~m}$ & $1.72 \mathrm{~m}$ \\
\hline VPL 99\% & $9.98 \mathrm{~m}$ & $10.33 \mathrm{~m}$ & $9.98 \mathrm{~m}$ & $10.39 \mathrm{~m}$ & $10.43 \mathrm{~m}$ \\
\hline $\begin{array}{c}\text { Continuity } \\
\text { Risk }\end{array}$ & $<8 \cdot 10^{-6}$ & $<8 \cdot 10^{-6}$ & $<8 \cdot 10^{-6}$ & $5.8 \cdot 10^{-3}\left({ }^{1}\right)$ & $7.1 \cdot 10^{-3}\left({ }^{1}\right)$ \\
\hline $\begin{array}{c}\text { Availability } \\
\text { (PL < AL) }\end{array}$ & $99.89 \%$ & $99.85 \%$ & $99.89 \%$ & $99.17 \%$ & $99.45 \%$ \\
\hline AER & $\mathrm{N} / \mathrm{A}$ & 0 & $\mathrm{~N} / \mathrm{A}$ & $0.5 \%$ & $0.3 \%$ \\
\hline
\end{tabular}

Table 8 - Summary of SBAS performance with and without EC-Schnorr and TESLA authentication, for cases with $\mathrm{PER}=0$ and $\mathrm{PER}=10^{-3}$

As general observations, the table shows that, when there is no authentication the PER $=0$ and $\mathrm{PER}=10^{-3}$ perform almost the same. This is expected, as message losses from the $\mathrm{PER}=10^{-3}$ case are very unlikely to last more than 1 second, and therefore VPE/VPL, availability and continuity remain unaffected. The table also shows a better performance of the L1/L5 case, with significantly lower VPE/VPLs and a higher continuity and availability, which remains above $99 \%$ after adding authentication in all cases.

Accuracy (VPE 95\%) is only slightly degraded by a few centimeters due to authentication. Consequently, VPLs $99 \%$ increase between $20 \mathrm{~cm}$ and around $1 / 2$ a meter, depending on the case.

As regards continuity, when $\mathrm{PER}=0$, the impact of authentication is negligible, but there is a high impact in all cases when message losses are taken into account: continuity risk rises up to $7.7 \cdot 10^{-3}$ for L1 EC-Schnorr and a similarly high values for other cases. This is due to the fact that every message loss, which otherwise would have a minimal performance impact, leads to a failed authentication, which in turn leads to dropping all the messages between authentications, and a continuity event. If the receiver can assume (in combination with other indicators) that it can coast until the next authentication, or extra message recovery or redundancy is added, the continuity requirement can be maintained, as shown in (Enge \& Walter, 2014). Note also that SBAS MOPS requires two SBAS links, and this redundancy, which is not considered in our analysis, provides some extra margin.

As regards availability, some degradation can be observed due to multiple factors: first, the fact that the authentication schemes used more than $20 \%$ of the bandwidth makes the PLs grow more often above the AL. This is particularly visible in L1, and has no availability impact in L5, given that dual-frequency PLs are sufficiently below AL. This effect in L1 will be mitigated if a lower bandwidth is consumed by authentication, or the $\mathrm{Q}$ channel is used. When message losses are considered, the bandwidth effect is combined with the authentication failures due to one message 
lost, which lowers availability down to $99.2 \%$ for TESLA L1, to $98.8 \%$ for EC-Schnorr L1, and down to $99.4 \%$ and $99.1 \%$ for each scheme in L5.

\section{CONCLUSIONS AND RECOMMENDATIONS}

This article has presented different SBAS authentication schemes and their performance if introduced in SBAS, using EGNOS as a reference. The data authentication schemes proposed by the EAST (EGNOS Authentication Security Test-bed) project partners, presented in this article, are standard implementations of EC-Schnorr digital signatures and TESLA. The work has also analyzed authentication data integrated in the L5 message sequence (I channel), L1 message sequence (I channel), or Q channel of either signal. When in the I channel, authentication fully occupies a given number of messages, without modifying the others. When in the Q channel, the proposed implementations occupy the full data stream. Interpretation of Time To Alert is a relevant design driver: if the 6-second TTA requirement applies to authentication, the use of the Q channel may be required. If the receiver can coast for a longer time without authentication, the I channel may be an option. The time between authentications, which drives the authentication latency, must be designed according to this assumption.

The proposed schemes in the I channel are very bandwidth-intensive, so as to bring authentication latency close to TTA. EC-Schnorr requires three (128-bit security) SBAS messages to transmit the digital signature and some overhead bits for overall status and over-the-air rekeying. TESLA can fit in 1 message, but it requires two messages for every authentication, one for the MAC and another for the key. Using around 20\% bandwidth, EC-Schnorr occupies three messages every 18 seconds, while TESLA one message every 6 seconds, but with a latency of 12 seconds. On the other hand, the proposed Q-channel implementations can yield a latency below the 6-second TTA. While a 50$50 \%$ power sharing is suboptimal, other schemes as $75 \%-25 \%$ or $80-20 \%$ for I-Q, with a lower coding rate in the $\mathrm{Q}$ channel, are more advisable.

The impact of adding authentication to L1-I and L5-I has been assessed for the two schemes in an EGNOS performance simulator, based on a reference scenario lasting 24 hours, and composed of real SBAS and GNSS data and a realistic EGNOS message scheduler, which was altered by the simulator to accommodate the authentication information. SBAS performance can be affected due to the bandwidth use and authentication failures. Accuracy is only slightly degraded by $4-8 \mathrm{~cm}$ for L1 and $6 \mathrm{~cm}$ for L5. VPLs increase is $20-55 \mathrm{~cm}$ for L1, and $35-45 \mathrm{~cm}$ for L5. However, there is a high impact in continuity as, with the current schemes and assumptions, every message loss leads to a failed authentication, which in turn leads to a continuity loss. Continuity impact can be minimized by minimizing AER due to message loss, for example by adding message recovery or redundancy. Some availability degradation can be observed due to the bandwidth occupation, when PLs exceed $\mathrm{AL}$ in L1, and also due to the continuity losses.

Therefore, adding SBAS authentication data in the I channel of both L1 and L5 seems a priori feasible. This assertion takes into account that the proposed schemes are just preliminary and highly bandwidth intensive, assumes that impact in continuity can be mitigated, and assumes that the receiver can coast beyond the 6-second TTA during an approach. Alternatively, transmitting authentication data in the Q channel can be an option: with an appropriate power apportionment and coding scheme, authentication data can be transmitted by only shifting less than $1 \mathrm{~dB}$ of power from the I channel to the $\mathrm{Q}$ channel. These considerations leave aside the impact in ground and space infrastructure, which may also be a significant driver of SBAS evolutions.

We can conclude that SBAS authentication seems a useful mitigation barrier against some spoofing threats, both by itself and in combination with other receiver-based measures, and there are various 
options by which it could be added to future standards. These options should be refined and tradedoff in due time.

\section{Acknowledgements}

The authors would like to thank Dr. Todd Walter and Dr. Juan Blanch and the anonymous reviewers for their useful comments in the preparation of this manuscript.

\section{REFERENCES}

Becker, G. T., Lo, S., Lorenzo, D. D., Qiu, D., Paar, C., \& Enge, P. (2009). Efficient authentication mechanisms for navigation systems - a radio-navigation case study. ION GNSS. Savannah.

Calle, D., Cancela, S., Carbonell, E., Rodríguez, I., Tobías, G., \& Fernández-Hernández, I. (2016). First Experimentation Results with the Full Galileo CS Demonstrator. ION GNSS+ 2016. Portland, OR.

Caparra, G., Ceccatto, S., Sturaro, S., \& Laurenti, N. (2017). A key management architecture for GNSS open service Navigation Message Authentication. ENC. Lausanne, $\mathrm{CH}$.

Caparra, G., Sturaro, S., Laurenti, N., \& Wullems, C. (2016). Evaluating the Security of One-way Key Chains in TESLA-based GNSS Navigation Message Authentication Schemes. International Conference for Localisation and GNSS (ICL-GNSS 2016). Barcelona.

Chiara, A. D., Broi, G. D., Pozzobon, O., Sturaro, S., Caparra, G., Laurenti, N., . . . Chatre, E. (2016). Authentication Concepts for Satellite-Based Augmentation Systems. Proceedings of the 29th International Technical Meeting of The Satellite Division of the Institute of Navigation (ION GNSS+ 2016). Portland, Oregon.

Enge, P., \& Walter, T. (2014). Digital Message Authentication for SBAS (and APNT). ION GNSS+ 2014. Tampa, FL.

Enge, P., Walter, T., \& Shallberg, K. (2017). Detection of GPS Spoofing - Working Group 2 of RTCA Special Committee 159.

ENISA. (2013). Algorithms, Key Sizes and Parameters Report - recommendations.

European Commission . (2017). COMMISSION IMPLEMENTING DECISION (EU) 2017/224 of 8 February 2017 (CS Implemeting Act).

European GNSS Agency. (2014). EGNOS Data Access Service (EDAS) Service Definition Document v2.1.

FAA Navigation Team. (2011). GPS Privacy Jammers and RFI at Newark Navigation Team AJP652 Results.

Fernández-Hernández, I. (2014). GNSS Authentication: Design Parameters and Service Concepts. Proceedings of the European Navigation Conference.

Fernández-Hernández, I., Rijmen, V., Seco-Granados, G., Simón, J., Rodríguez, I., \& Calle, J. D. (2016). A Navigation Message Authentication Proposal for the Galileo Open Service. NAVIGATION, the Journal of the Institute of Navigation.

Hünerbein, K. v., \& Lange, W. (2015). Receivers, Real Life Evidence for Spoofing and Jamming of GNSS. CERGAL. ESOC, Darmstadt.

International Civil Aviation Organisation. (2006). International Standards and Recommended Practices - Annex 10 - Aeronautical Telecommunications - Vol 1 - Radio Navigation Aids. ICAO publications.

International Standards Organisation. (2009). ISO/IEC 14888-3. Information technology - Security techniques - Digital signatures with appendix - Part 3 - Discrete logarithm based mechanisms - Ammendment 1. International Standards Organisation.

International Telecommunication Union. (2015). Recommendation ITU-R P.681-8,Propagation data required for the design of Earth-space land mobile telecommunication systems. ITU-R.

Kerns, A. J., Wessons, K., \& Humphreys, T. (2014). A Blueprint for Civil GPS Navigation Message Authentication. Proceedings of Position, Location and Navigation Symposium. Monterey: PLANS 2014, 2014 IEEE/ION.

Lin, S., \& Costello, D. J. (2004). Error control coding (Vol. 2). Englewood Cliffs. Prentice Hall. 
Lo, S. C., \& Enge, P. K. (2010). Authenticating aviation augmentation system broadcasts. Position Location and Navigation Symposium (PLANS), 2010 IEEE/ION (pp. 708-717). .

Margaria, D., Motella, B., Anghileri, M., Floch, J. J., Fernández-Hernández, I., \& Paonni, M. (2017, September). Signal Structure-Based Authentication for Civil GNSSs - Recent solutions and perspectives. IEEE Signal Processing Magazine.

National Institute of Standards and Technology. (2008). FIPS PUB 198-1: The Keyed-Hash Message Authentication Code (HMAC).

National Institute of Standards and Technology. (2012). FIPS PUB 180-4: Secure Hash Standard (SHS).

Perrig, A., Canetti, R., Tygar, J., \& Song, D. (2000, May). Efficient Authentication and Signing of Multicast Streams over Lossy Channels. IEEE Symposium on Security and Privacy, pp. 5673.

Psiaki, M. L., \& Humphreys, T. E. (2016). GNSS Spoofing and Detection. Proceedings of the IEEE , 104(6).

RTCA SC-159. (2006). MOPS WAAS - Minimum Operational Performance Standards for Global Positioning System Wide Area Augmentation System Airborne Equipment- DO229D (with Change 1, Feb 2013).

SBAS Interoperability Working Group Issue 1 Revision 3 April 2016. (April 2016). SBAS L5 DFMC Interface Control Document (SBAS L5 DFMC ICD) Issue 1 Revision 3.

Scott, L. (2003). Anti-Spoofing \& Authenticated Signal Architectures for Civil Navigation Systems. ION GPS.

Walker, P., Rijmen, V., Fernandez-Hernandez, I., Simón, J., Calle, D., Pozzobon, O., \& SecoGranados, G. (2015). Galileo Open Service Authentication: A Complete Service Design and Provision Analysis. Proceedings of ION GNSS+ 2015. Tampa.

Wesson, K., Rothlisberger, M., \& Humphreys, T. (2012, February). Practical Cryptographic Civil GPS Signal Authentication. NAVIGATION, The Journal of the Institute of Navigation.

Wullems, C., Pozzobon, O., \& Kubik, K. (2005). Signal Authentication and Integrity Schemes for Next Generation Global Navigation Satellite Systems. Proceedings of the European Navigation Conference.

Yuan, M., Lv, Z., Chen, H., Li, J., \& \& Ou, G. (2017). An Implementation of Navigation Message Authentication with Reserved Bits for Civil BDS Anti-Spoofing. China Satellite Navigation Conference. Singapore. 